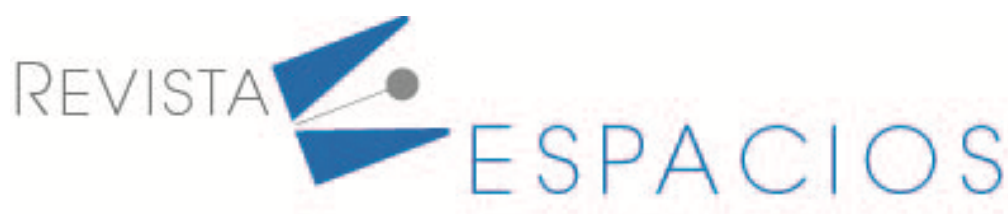

\title{
Percepción de estudiantes universitarios colombianos sobre el efecto del confinamiento por el coronavirus, y su rendimiento académico
}

\section{Perception of Colombian university students on the effect of confinement by the coronavirus, and their academic performance}

\author{
VALDIVIESO MIRANDA, Margoth A. ${ }^{1}$ \\ BURBANO PANTOJA, Víctor M. ${ }^{2}$ \\ BURBANO VALDIVIESO, Ángela S. ${ }^{3}$
}

\begin{abstract}
Resumen
Este estudio explora la percepción de un grupo de estudiantes universitarios colombianos sobre el efecto del coronavirus y el confinamiento sobre su rendimiento acdémico (RA). La metodología fue cuantitaiva con diseño transversal de tipo exploratorio-descriptivo; la información se recolectó mediante una encuesta aplicada a 85 estudiantes, el análisis estadístico fue efectuado con el software SPSS. Los resultados reflejaron que, la virtualidad, el estado de ánimo y la convivencia familiar afectaron su RA. Se concluyó que la cuarentena afectó el RA estudiantil.

Palabras clave: pandemia (covid-19), percepción, clases virtuales, convivencia, estado de ánimo, rendimeinto académico-cuarentena.
\end{abstract}

\begin{abstract}
This study explores the perception of a group of Colombian university students about the effect of the coronavirus and confinement on their academic performance (AR). The methodology was quantitative with a cross-sectional exploratory-descriptive design; the information was collected by means of a survey applied to 85 students, the statistical analysis was carried out with the SPSS software. The results reflected that virtuality, mood and family life affected their AR. It was concluded that the quarantine affected the student RA.

Key words: pandemic (covid-19), perception, virtual classes, coexistence, mood, academic performance-quarantine.
\end{abstract}

\section{Introducción}

Históricamente han existido muchas pandemias como se observa en la Figura 1 (Trujillo, 2020) y en la actualidad hemos sido afectados por una enfermedad muy silenciosa que se ha constituido en pandemia, según la Organización Mundial de la Salud (OMS, 2020a), la COVID-19 es una enfermedad infecciosa causada por el

\footnotetext{
${ }^{1}$ Licenciada en Matemáticas, Magíster en Ciencias-Estadística. Escuela de Matemáticas y Estadística. Filiación: Universidad Pedagógica y Tecnológica de Colombia, calle 48 No. 8-48 Tunja, Colombia. Correo margoth.valdivieso@uptc.edu.co

${ }^{2}$ Magíster en Estadística, Doctor en Ciencias de la Educación. Escuela de Matemáticas y Estadística. Universidad Pedagógica y Tecnológica de Colombia, calle 48 No.8-48 Tunja, Colombia victor.burbano@uptc.edu.co

${ }^{3}$ Tecnóloga en programación de sistemas informáticos. Bióloga. Filiación: Universidad Pedagógica y Tecnológica de Colombia. Investigadora Grupo Interdisciplinario en Ciencias GICI. Correo angela.burbano@uptc.edu.co
} 
coronavirus que se descubrió recientemente, tanto el virus como la enfermedad que provoca eran desconocidos antes de que estallara el brote en Wuhan (China) en diciembre de 2019. La propagación del virus ha sido rápida y los gobiernos apoyados con el personal de la salud han tratado de controlar su expansión y efectos letales. A la fecha del 20 de septiembre de 2020, los medios de comunicación y la Universidad Johns Hopkins habían notificado que a nivel mundial, se tenían más de 32.76 millones de casos de COVID-19 y se habían perdido 957.189 vidas. En este contexto, el coronavirus se ha constituido en un enemigo invisible y silencioso con el cual hay necesidad de empezar a convivir por cuanto ha llegado para quedarse (Ortíz, Herrera \& De La Torre, 2020). En estas circunstancias, mientras dure esta pandemia, se espera que las personas sientan miedo, ansiedad, angustia, irritabilidad, estrés y enojo, recuerdo de traumas, dificultades para la concentración y/o problemas en el sueño, al desconocer cuando el virus las podrá acatar (Johnson, Saletti y Tumas, 2020).

Figura 1

Historia de las pandemias

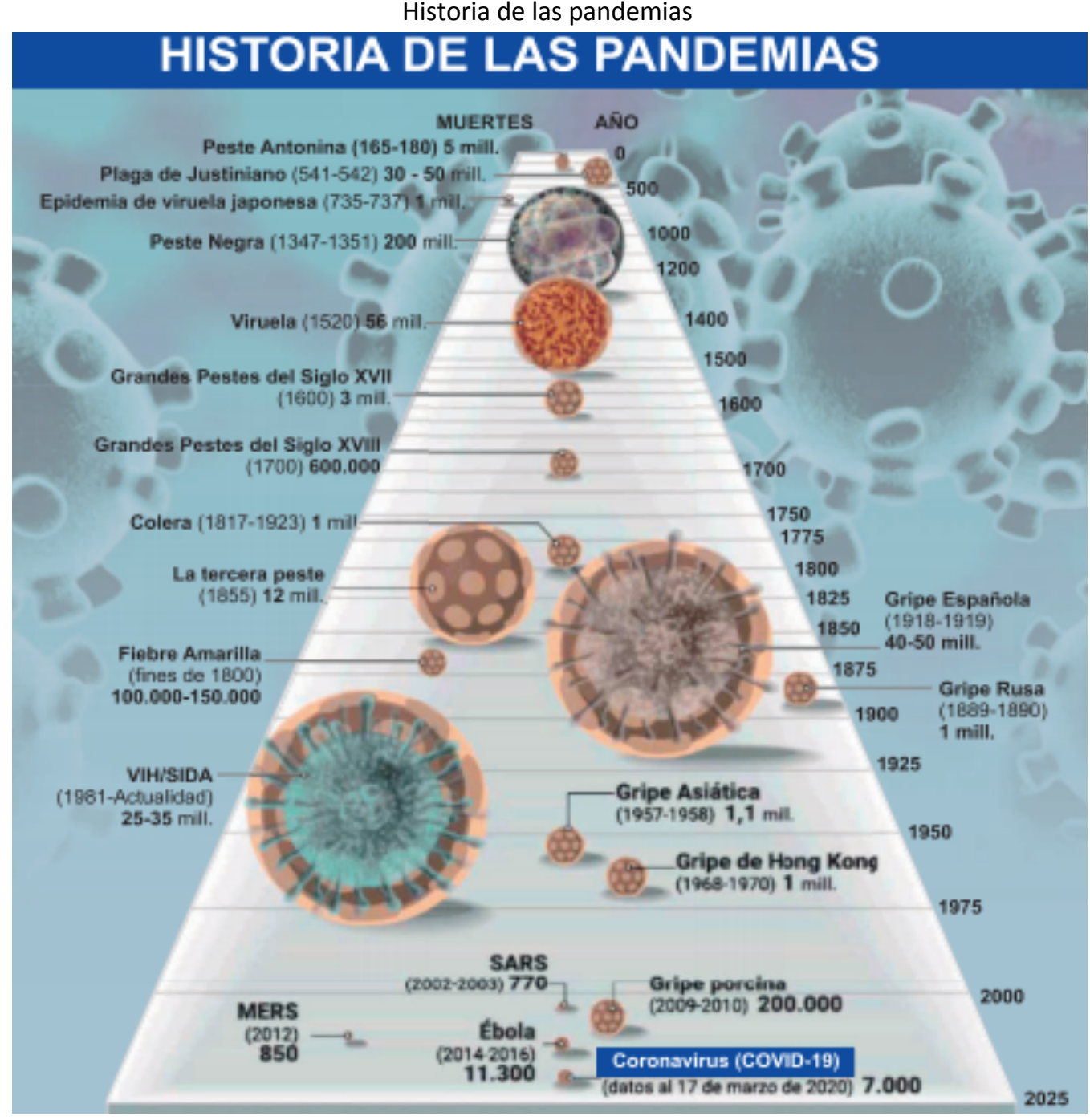

En: Organización Mundial de la Salud y Enciclopedia Británica 2020, referenciadas por Trujillo (2020, p. 148)

Cada pandemia trae consigo varias consecuencias, entre ellas se pueden considerar las económicas, educativas, psicológicas, políticas, familiares y sociales, entre otras; particularmente lo relacionado con el aislamiento social forzando a permanecer en cuarentena durante largos lapsos de tiempo. Así como en su momento, el capitalismo instauró la premisa "todo es posible", "todo está permitido", ahora el coronavirus exige regulaciones, privaciones y límites en las formas de vivir de las personas, cambios en las maneras de actuar de los grupos sociales y 
controles socioculturales (Schor, 2020). En este sentido, en un estudio impulsado por la Facultad de Ciencias Sociales de la Universidad Nacional de Córdoba en Argentina (2020), se muestra que la pandemia ha generado un sin número de preocupaciones e intranquilidades en la vida de las personas, en su subsistencia, en las dificultades para seguir trabajando o para que sus hijos puedan continuar sus estudios bajo propuestas educativas improvisadas y centradas en los medios virtuales, mucho más, cuando miles de padres de familia no tienen las condiciones para sostenerlos, comprarles los computadoras y pagarles los accesos a internet.

Por otra parte, se puede ver la situación como un fenómeno incierto que también puede ofrecer oportunidades; el aislamiento social derivado de la COVID-19, en varios casos ha fomentado la creatividad, el dinamismo y la autonomía de los estudiantes en un contexto donde el uso de las Tecnologías de la Información y la Comunicación (TIC) y los videotutoriales están transformando la educación tradicional (Millán, 2020). Sin embargo, el aislamiento social preventivo también ha impactado el estilo de vida de todos los estudiantes a nivel mundial (Sudriá, Andreatta, y Defagó, 2020), en unos casos ha afectado su estado emocional, físico y social, en otros su rendimiento académico y sus costumbres habituales; la presencia del Covid 19 les ha acrecentado su percepción sobre una amenaza latente para su salud y el bienestar de los demás miembros de su familia, con impactos en diversos aspectos de su vida (Sandman, 1993; Costa \& López, 2020); en este sentido, conviene explorar las percepciones del estudiante universitario en referencia a su rendimiento académico asociado con la virtualidad, la convivencia familiar y el estado de ánimo durante la cuarentena o los periodos de confinamiento en el hogar.

Para menguar los efectos del Covid 19, en Colombia, el gobierno central declaró la emergencia sanitaria con la expedición de varios decretos tendientes a disminuir la velocidad del contagio y a recuperar a los contagiados; estos decretos empezaron a ser aplicados el 16 de marzo de 2020; desde esa fecha, se han desarrollado diversas estrategias lideradas por el presidente de la república, el Ministerio de Salud colombiano y los gobiernos tanto departamenteles como municipales, con el propósito de evitar la propagación del contagio por coronavirus a lo largo y ancho del territorio nacional y con la participación ciudadana, hacerle frente común a la pandemia (Membiela, Sánchez \& Pena, 2019); para esto, se han establecido medidas como la adecuación de la infraestructura hospitalaria, el distanciamiento social, los protocolos de bioseguridad, periodos de confinamiento para trabajadores, profesores y estudiantes de manera sectorizada o regionalizada, ayudas económicas a los hogares de bajos ingresos, cierres parciales de los medios de transporte, entre otros. Los periodos de confinamiento produjeron una parálisis total pero temporal del sistema productivo colombiano, el cierre de las universidades y colegios, con el compromiso de que las clases se continuaran ofreciendo desde los hogares, por medio de herramientas virtuales (Martínez \& Garcés, 2020). En este contexto, miles de estudiantes universitarios debieron recibir sus clases desde su casa para minimizar el riesgo de contagio masivo, pero la pregunta es ¿los estudiantes, padres de familia y profesores universitarios estaban preparados para atender estas eventualiades?

La respuesta no es inmediada, pero los padres de familia debieron hacer esfuerzos gigantescos para aprovisionar a sus hijos de las herramientas tecnológicas para que pudieran recibir sus clases virtuales; por otra parte, la universidad también debió hacer grandes esfuerzo de tipo económico y logístico para apoyar a los estudiantes de bajos recursos económicos con computadores y dipositivos móviles para que tuvieran conectividad y puedieran recibir sus clases virtuales; por su parte los profesores universitarios debieron cambiar su rol, actualizarse en el uso de medios virtuales y estrategias propias de la educación virtual para atender al estudiantado y desarrollar sus microcurrículos por medio de metodologías basadas en el teletrabajo (Caridad, Castellano \& Cardeño, 2019). Es conveniente mencionar que, las universidades públicas colombianas estaban parcialmente preparadas para enfrentar una situación tan difícil e incierta como la generada por el coronavirus, a pesar de que han de contar con un plan de riesgos y atención de emergencias; una de las decisiones que se debió tomar fue la de adoptar las medidas decretadas por el gobierno nacional y enviar a sus docentes, estudiantes y trabajadores a confinamiento hasta que se supere la emergencia sanitaria; esta situación obligó a 
que los estudiantes y profesores trabajaran con metodologías propias de la educación a distancia y virtual (Mendoza, Burbano \& Valdivieso, 2019), con el fin de mantener el derecho a la educación de los estudiantes y de no cancelar su semestre académico; este nuevo desafío desencadenó diversas afectaciones en la vida estudiantil, generando distintas reacciones, maneras de percibir y asumir sus compromisos académicos.

Los aspectos mencionados también se presentaron en el ámbito de la Universidad Pedagógica y Tecnológica de Colombia (UPTC), en su entorno todo cambió, de un día para otro se asumió una gran responsabilidad, pasar de la educación presencial a la educación virtual; esta determinación ha generado impactos colaterales en los procesos de formación de su población estudiantil (Díaz, Prados, Canos \& Martínez, 2020, UNICEF Argentina, 2020). En general, estos cambios fueron asumidos por la comunidad educativa universitaria de la UPTC de forma gradual y en particular por los estudiantes, quienes son consientes de que están viviendo un nuevo mundo, matizado por el riesgo al contagio y el desafío por asimilar sus aprendizaje a través de medios virtuales; aunque los estudiantes son quienes han estado a la vanguardia de las nuevas tecnologías, los celulares y la web (Pincay, 2018), aún no asimilaban de forma plena el escenario de recibir clases virtuales mediadas con las Tecnologías de la Información y las Comunicaciones (TIC); además, un buen número de estudiantes carecía de recursos tecnológicos como el computador, la conexión a internet en su casa o el celuar con planes de datos, destinados al desarrollo de sus actividades académicas en estado de aislamiento (cuarentena) desde su hogar.

Las eventualidades mencionadas anteriormente, hicieron que la UPTC implementara algunos planes de contingencia para solucionar el problema de conectividad de más de 2000 estudiantes, generar espacios de capacitación en herramientas virtuales a docentes y realizar acciones de planeación curricular para la virtualidad, de modo que, el inicio de las actividades académicas (procesos de enseñanza-aprendizaje virtuales) debieron postergarse hasta el mes de mayo de 2020. Una vez había empezado el semestre y con los estudiantes universitarios en cuarentena (confinamiento), emergen en ellos situaciones familiares, sociales, de estado de ánimo, de convivencia e interacción virtual que, según su percepción pueden afectar su rendimiento académico; en este contexto, resulta pertinente indagar sobre tales afectaciones y generar espacios de reflexión que permitan mejorar los escenarios de aprendizaje para los estudiantes de la UPTC.

En este marco de referencia, es conveniente mencionar que, por lo general los estudiantes de la UPTC provienen de diversos puntos de Boyacá en Colombia y de muchas regiones del país, habitualmente desarrollaban su vida académica en las instalaciones del campus universitario, en el cual, contaban con el soporte tecnológico de la UPTC en su infraestructura física para el desarrollo de sus actividades académicas, deportivas, de bienestar, restaurante, bibliotecas, entre otras, tenían una buena convivencia con sus compañeros, sus docentes y el entorno administrativo que trabajaba en el campus; para varios estudiantes, su lugar de descanso y recogimiento al final de su jornada se encontraba entre las residencias universitarias, los apartaestudios, los lugares de arrendamiento, las casas familiares o los propios hogares. El confinamiento los obligó a retornar a sus lugares de orígen, donde no disponen de los recursos mencionados o hay fallas en el servicio de internet (conectividad), esta situación e imprevistos posiblemente afectaron la dinámica de sus clases virtuales, su rendimiento académico y su estado emocional. Los escenarios descritos anteriormente, proporcionaron elementos de reflexión y justificaron la necesidad de realizar este estudio.

\section{Metodología}

Este estudio se desarrolló con un enfoque cuantitativo de investigación y un diseño de corte transversal, en este diseño la información se recogió en un lapso determinado del tiempo (Sampieri, 2018); la metodología se focalizó en un análisis de tipo exploratorio-descriptivo, el cual permitió la recolección, organización, representación e interpretación de los resultados (Valdivieso, 2011), con el fin de obtener conclusiones que apoyen la toma de decisiones en el contexto universitario en el ámbito estudiantil. Además, se realizaron inferencias para 
determinar la asociación entre algunas variables de interés (Ondé, 2017; Mulaik, 2009), cuando aquellas son variables categóricas o cualitativas (Tinoco, 2008).

Para esta investigación se seleccionó una muestra no probabilística conformada por 85 estudiantes universitarios de la UPTC de la Ciudad de Tunja-Colombia de varios programas, semestres y cursos diversos, de los cuales 39 (45.88\%) fueron hombres y 46 (54.12\%) mujeres, los participantes presentaron edades desde los 17 hasta los 38 años. Los datos (información) fueron recolectados por medio de una encuesta con consentimiento informado previo. De acuerco con la Ley 2266 del año 2008, se hizo conocer a los participantes que los datos recogidos se manejarían de forma confidencial, usándolos de manera ética (Habeas data), dentro de un contexto académico o investigativo.

Entre las características (variables) incluidas en la encuesta están: convivencia en el núcleo familiar, estado de ánimo, tareas del hogar antes y durante de la cuarentena, acuerdo o desacuerdo con el proceso enseñanzaaprendizaje por medios virtuales, la virtualidad y la adquisición de nuevos conocimientos, la virtualidad y el afianzamiento de conocimientos, afectación del rendimiento académico por la convivencia familiar, afectación del rendimiento académico por confinamiento, estrato socioeconómico, lugar donde el estudiante habitaba antes y durante la cuarentena, sexo, edad, semestre al que pertenecía, entre otras. Para valorar el estado de ánimo se utilizó la escala EVEA, la cual incluye cuatro estados de ánimo generales: ansiedad, ira-hostilidad, tristeza-depresión, y alegría (Sanz, 2001; Del Pino, Peñate \& Bethencourt, 2010); también se involucraron otros tipos de respuesta como: incertidumbre, desmotivación, estado de ánimo neutro o normal, entre otros.

El procesado de los datos se elaboró por medio del software SPSS en su versión de prueba a 30 días, disponible en internet. Con base en las preguntas de la encuesta, se efectuó un análisis exploratotio-descriptivo basado en tablas de porcentajes y salidas del SPSS, el cual fue complementado con exégesis textual (Strauss y Corbin, 1998; Burbano, Valdivieso \& Burbano, 2020); además, se emplearon tablas de contingencias que permitieron interpretar los hallazgos relacionados con el estado de ánimo estudiantil durante la pandemia, el confinamiento y el rendimiento académico del estudiante universitario de la UPTC, tal rendimiento académico fue el resultado de su interacción con los materiales digitales de aprendizaje y las tutorías recibidas de sus docente. Finalmente, la raíz epistemológica de la percepción del riesgo se sustentó en la fenomenología social focalizada en la epojé o descripción de una serie de indicadores convergentes en la inconmensurabilidad, impredecibilidad e incontrolabilidad de eventos considerados amenazas por su desconocimiento (Carreón, 2018; Nájera, Aguayo, Guillén \& Lirios, 2020), los cuales son elementos característicos de la pandemia por coronavirus.

\section{Resultados}

A continuación se presentan los principales hallazgos de este estudio, los cuales se han organizado en tres secciones: la primera referida a los aspectos contextuales, la segunda a las variables específicas asociadas con el objeto de estudio y la tercera, al análisis de asociación entre las variables convivencia familiar, estado de ánimo y virtualidad con respecto al rendimiento académico.

\subsection{Hallazgos relacionados con los aspectos contextuales de los estudiantes universitarios}

De los estudiantes encuestados, el $90.6 \%$ vivía en la ciudad de Tunja-Boyacá antes de la pandemia y el resto en ciudades cercanas, aproximadamente un $36 \%$ de los estudiantes universitarios vivían solos en apartaestudios o habitaciones en arrendamiento, un $39 \%$ vivía con su propia familia y el restante $25 \%$ estaba en casas de familia, residencias estudiantiles, amigos, familiares como abuelos, hermanos, tías o primos, o en sus hogares conformados por su pareja e hijos. Una vez declarada la cuarentena (confinamiento) el $43.55 \%$ de los estudiantes de la UPTC que vivían en Tunja tuvieron que emigrar a sus lugares de origen, ubicados en municipios como Aguazul, Barbosa, Bogotá, Chiquinquirá, Chocontá, Corraleñas, Duitama, Motavita, Nobsa, Pacho-Cundinamarca, 
Paipa, Ramiriquí, Sáchica, San Mateo, Santa María, Santana, Sativa Norte, Sogamoso, Tasco, Tibasosa, Tinjacá, Tipacoque, Tuta, Ventaquemada, Villa de Leyva y Villavicención. En este contexto se evidencia la variabilidad del lugar de procedencia de los estudiantes, por ello el estado de ánimo de los estudiantes se ve afectado por la convivencia familiar durante la cuarentena, la cual ha implicado mezclar las actividades hogareñas y el estudio de las asignaturas de su carrera profesional a través de medios virtuales.

La edad promedio del grupo de estudio fue de $20.77 \pm 3.11$ años, donde el valor \pm 3.11 corresponde a la desviación estándar (DE), la edad mínima fue de 17 años y la máxima de 38; se obtuvo un coeficiente de variación de $\mathrm{VC}=3.1 / 20.77=0.1497=14.97 \%$, este porcentaje indica que los estudiantes resultaron levemente heterogéneos, al presentarse algunas edades próximas a los datos extremos de 17 o 38 años. En referencia al estrato socioeconómico, un $17.6 \%$ pertenecen al estrato uno, el $65.9 \%$ al estrato dos, el $14.1 \%$ al estrato tres y el $2.4 \%$ al estrato cuatro. En lo referente al semestre de su carrera profesional que estaban cursando se estableció que, los estudiantes encuestados estaban ubicados desde el tercero hasta el décimo semestre y se distribuyeron de la siguiente forma: 22 (25.9\%) en el tercer semestre, 37 (43.5\%) en el cuarto, 8 (9.4\%) en el quinto, $3(3.5 \%)$ en sexto, $7(8.2 \%)$ en séptimo, 5 (5.9\%) en octavo, $1(1.2 \%)$ en noveno y 2 (2.4\%) en décimo semestre; estos resultados indican que existe una prevalencia mayor de estudiantes cursando el tercero o cuarto semestre en la UPTC.

\subsection{Variables específicas asociadas con el objeto de estudio}

En lo referente a la convivencia en el núcleo familiar se determinó que, antes de la cuarentena (confinamiento), para el $2.4 \%$ de los estudiantes de la muestra la convivencia familiar era mala, para el $17.6 \%$ era regular, para el $51.8 \%$ era buena y para el $28.2 \%$ era excelente. Durante el periodo de confinamiento, para el $3.5 \%$ de los estudiantes de la muestra la convivencia familiar se tornó mala, para el $18.8 \%$ fue regular, para el $56.5 \%$ fue buena y para el $21.2 \%$ resultó excelente (ver Tabla 1 ). Estos hallazgos reflejan que, con el retorno de los estudiantes universitarios a sus lugares de origen y al seno de sus hogares, la convivencia familiar se deterioró moderadamente en las categorías de malo y excelente, porque debían atender simultáneamente a sus estudios a través de educación virtual y colaborar con algunas tareas propias del hogar o las actividades del campo; es decir, debían extender sus jornadas diarias para atender a sus estudio y a otras labores.

Tabla 1

Convivencia familiar antes y durante la cuarentena

\begin{tabular}{lcc}
\hline & Antes & Durante \\
\hline Mala & $2.4 \%$ & $3.5 \%$ \\
Regular & $17.6 \%$ & $18.8 \%$ \\
Buena & $51.8 \%$ & $56.5 \%$ \\
Excelente & $28.2 \%$ & $21.2 \%$ \\
\hline
\end{tabular}

Fuente: elaboración propia

Una exploración de la convivencia familiar de los estudiantes universitarios por género reflejó que, en las estudiantes mujeres los niveles malos de convivencia familiar se incrementaron en un $3 \%$ aproximadamente durante el confinamiento; en cambio en los hombres ni antes ni durante la cuarentena su convivencia familiar fue mala. Además, se observa que aproximadamente el 18.2\% de los estudiantes del grupo de estudio perciben que mantienen una convivencia regular con su familia; por otra parte, durante la pandemia mejoró en un $4 \%$ la categoría de buen nivel de conveviencia familiar y se deterioró en un $7 \%$ el nivel excelente, entre las causas de este descenso se encuentra la desconcentración en el momento de asistir a clases virtuales y a la poca colaboración de los miembros de la familia cuando los estudiantes debían desarrollar sus actividades académicas. Al cruzar la convivencia familiar con la edad también se encontró que, los estudiantes mayores de 24 años tenían y conservaban la buena o excelente convivencia con sus familias. 
En cuanto al estado de ánimo se estableció que, antes de la cuarentena (confinamiento), el $11.8 \%$ de los estudiantes universitarios de la muestra presentaba estados de ansiedad, el 1.2\% tenía ira 4 hostilidad, el $7.0 \%$ sufría de tristeza $\theta$ depresión, el 71.8\% sentía alegría, el 7.0\% era neutro y el 1.2\% pasó por todos los anteriores estados emocionales. Durante el periodo de confinamiento algunos de estos estados tuvieron una abrupta variabilidad y en otros fue leve; el $45.9 \%$ de los estudiantes universitarios de la muestra presentaba estados de ansiedad, el 3.5\% tenía ira u hostilidad, el 23.5\% sufría de tristeza ө depresión, el $18.9 \%$ sentía alegría, el 3.5\% era neutro y el $4.9 \%$ pasó por todos los mencionados estados emocionales (ver Tabla 2). Estos hallazgos evidenciaron que, con el retorno de los estudiantes universitarios a sus lugares de origen y al seno de sus familias, la ansiedad se incrementó fuertemente en un 34.1\% al pasar del $11.8 \%$ al $45.5 \%$, quizá porque debían atender sus estudios a través de educación virtual, colaborar con algunas tareas propias del hogar o las noticias sobre el coronavirus les generaban desesperación y angustia; la alegría disminuyó en un $52.9 \%$ al pasar del $71.8 \%$ al $18.9 \%$, atribuible a las labores adicionales que les significaba hacer tareas académicas, estudiar para las evaluaciones y colaborar en el hogar; la tristeza $\bullet$ la depresión crecieron en un $16.5 \%$ al variar del $7.0 \%$ al $23.5 \%$. Los estados de ánimo referidos a la ira-hostilidad, neutro y el paso por todos los demás estados se afectaron levemente durante la cuarentena; sin embargo, esta situación es preocupante y debería atenderse de forma prioritaria con la intervención de la familia y la universidad.

Tabla 2

Estado de ánimo antes y durante la cuarentena

\begin{tabular}{lcc}
\hline & Antes & Durante \\
\hline Ansiedad & $11.8 \%$ & $45.9 \%$ \\
Ira-hostilidad & $1.2 \%$ & $3.5 \%$ \\
Tristeza-depresión & $7.0 \%$ & $23.5 \%$ \\
Alegría & $71.8 \%$ & $18.9 \%$ \\
Neutro & $7.0 \%$ & $3.5 \%$ \\
Paso por todos los estados & $1.2 \%$ & $4.7 \%$ \\
\hline
\end{tabular}

Fuente: elaboración propia

Sobre las tareas del hogar que con frecuencia debían realizar los estudiantes además de las actividades inherentes a su formación universitaria se encontró que, antes del confinamiento, el $37.6 \%$ de los estudiantes solamente hacía labores de aseo, el $23.6 \%$ hacía aseo y cocinaba, el $18.8 \%$ aseaba, cocinaba y lavaba la loza, el $11.8 \%$ aseaba, cocinaba, lavaba la loza y la ropa, el $8.2 \%$ aseaba, cocinaba, lavaba loza-ropa y hacía asepsia, entre otras. Durante el periodo de confinamiento estas tareas mostraron un comportamiento inverso para $11.8 \%$ de los estudiantes que solamente hacía labores de aseo, y para el $17.6 \%$ que hacía aseo y cocinaba; por otro lado se incrementó considerablemaente para los que aseaban, cocinaban y lavaban la loza en un 23.6\%; el $25.9 \%$ aseaba, cocinaba, lavaba la loza y la ropa, el $21.1 \%$ aseaba, cocinaba, lavaba loza-ropa y hacía asepsia, entre otras (ver Tabla 3). Estos resultados permitieron evidenciar que, con el retorno de los estudiantes universitarios a sus lugares de origen, se incrementaron labores estudiantiles habituales y tuvieron que soportar dos cargas adicionales: la interacción con sus profesores por medios virtuales para avanzar en sacar adelante su carrera profesional y la colaboración para realizar otras actividades propias del hogar, entre ellas las de asepsia o las del trabajo en el sector rural; estos factores están asociados con la ansiedad, la tristeza y la depresión las cuales a su vez influyen en el rendimiento académico de los estudiantes. 
Tabla 3

Tareas del hogar antes y durante la cuarentena

\begin{tabular}{lcc}
\hline & Antes & Durante \\
\hline Asear & $37.6 \%$ & $11.8 \%$ \\
Asear y cocinar & $23.6 \%$ & $17.6 \%$ \\
Asear, cocinar y lavar la losa & $18.8 \%$ & $23.6 \%$ \\
Asear, cocinar, lavar losa y ropa & $11.8 \%$ & $25.9 \%$ \\
Asear, cocinar, lavar losa-ropa y tareas de asepsia, otras & $8.2 \%$ & $21.1 \%$ \\
\hline \multicolumn{2}{r}{ Fuente elaboración propia. } \\
\hline
\end{tabular}

Fuente: elaboración propia.

En la Tabla 4 se presentan los resultados referidos a la percepción de los estudiantes sobre las variables: acuerdo o desacuerdo con el proceso enseñanza-aprendizaje por medios virtuales, la virtualidad posibilitó adquisición de nuevos conocimientos, la virtualidad permitió el afianzamiento de conocimientos, afectación del rendimiento académico por la convivencia familiar y afectación del rendimiento académico por confinamiento. Respecto a la primera, el $32.9 \%$ de los estudiantes estuvo de acuerdo con el proceso enseñanza-aprendizaje mediante herramientas virtuales y el $67.1 \%$ estuvo en desacuerdo, porque antes de la pandemia habían interactuado poco con las aulas virtuales disponibles en la UPTC; el 71.8\% considera que, la virtualidad si le posibilitó la adquisición de nuevos conocimientos y el $28.2 \%$ piensa que no; el $63.5 \%$ acepta que la virtualidad le permitió el afianzamiento de conocimientos y el $36.5 \%$ percibe que no; el $36.5 \%$ considera que la convivencia familiar durante la cuarentena si afectó su rendimiento académico y el $63.5 \%$ admite que no; finalmente, el $77.6 \%$ percibe que el confinamiento si afectó su rendimiento académico, en cambio el $22.4 \%$ considera que no (ver Tabla 4). Estos resultados proporcionan indicios de que el rendimiento académico se afectó principalmente por la cuarentena y la convivencia familiar; esta última le disminuyó el tiempo para realizar sus actividades académicas (trabajo independiente virtual) y la preparación de sus evaluaciones. Por consiguiente, el retorno de los estudiantes universitarios a sus lugares de origen, fue una solución para contener el contagio del coronavirus pero en detrimento de su rendimiento académico.

Tabla 4

Afectación del rendimiento académico estudiantil por el confinamiento, la vitualidad y la convivencia familiar

\begin{tabular}{lcc}
\hline & Sí & NO \\
\hline Está de acuerdo con el proceso enseñanza-aprendizaje por medios virtuales & $32.9 \%$ & $67.1 \%$ \\
La virtualidad le posibilitó la adquisición de nuevos conocimientos & $71.8 \%$ & $28.2 \%$ \\
La virtualidad le permitió el afianzamiento de conocimientos & $63.5 \%$ & $36.5 \%$ \\
La convivencia familiar le afectó su rendimiento académico & $36.5 \%$ & $63.5 \%$ \\
El confinamiento le afectó su rendimiento académico & $77.6 \%$ & $22.4 \%$ \\
\hline
\end{tabular}

Fuente: elaboración propia.

\subsection{Análisis de asociación entre variables}

En la Tabla 5 se indican los resultados de la asociación existente entre los niveles de convivencia familiar y la afectación del redimiento académico; los hallazgos evidencian que, a un $58.8 \%$ de los estudiantes del grupo quienes tuvieron una convivencia familiar buena o excelente, les afectó su rendimiento académico de forma positiva y a un $18.7 \%$ con estas mismas condiciones de convivencia les afectó su rendimiento académico de manera negativa. Por otro lado, a un $18 \%$ de los estudiantes del grupo quienes presentaron una convivencia familiar regular o mala también le afectó su rendimiento académico de forma positiva y a un 3.5\% que presentó convivencia regular le afectó su rendimiento académico de forma negativa. La prueba chi-cuadrado permitió 
establecer la existencia de una asociación moderada entre la conivencia familiar y la afectación del rendimiento académico ( $\mathrm{P}$-valor $=0.311>0.05)$.

Tabla 5

Asociación entre convivencia familiar y afectación del rendimiento académico (RA)

\begin{tabular}{|c|c|c|c|c|c|c|c|}
\hline & & & \multicolumn{4}{|c|}{ Convivencia familiar durante la cuarentena } & \multirow[t]{2}{*}{ Total } \\
\hline & & & Mala & Regular & Buena & Excelente & \\
\hline \multirow[t]{4}{*}{ RA } & Positivo & Count & 3 & 13 & 34 & 16 & 66 \\
\hline & & $\%$ of Total & $3,5 \%$ & $15,3 \%$ & $40,0 \%$ & $18,8 \%$ & $77,6 \%$ \\
\hline & Negativo & Count & 0 & 3 & 14 & 2 & 19 \\
\hline & & $\%$ of Total & $0,0 \%$ & $3,5 \%$ & $16,5 \%$ & $2,4 \%$ & $22,4 \%$ \\
\hline \multirow[t]{2}{*}{ Total } & & Count & 3 & 16 & 48 & 18 & 85 \\
\hline & $\%$ Total & & $3,5 \%$ & $18,8 \%$ & $56,5 \%$ & $21,2 \%$ & $100,0 \%$ \\
\hline
\end{tabular}

Fuente: elaboración propia.

En la Tabla 6 se indican los resultados de la asociación existente entre el estado de ánimo y la afectación del redimiento académico; los hallazgos permitieron establecer que, a un 34.1\% de los estudiantes del grupo quienes estaban ansiosos durante la cuarentena, sí les afectó su rendimiento académico, en cambio al $11.8 \%$ no les afectó; a un 21.2\% de los estudiantes del grupo quienes presentaron un estado de tristeza o depresión durante el confinamiento les afectó su rendimiento académico, mientras que al $32.4 \%$ no les afectó; a un $14.1 \%$ de quienes estaban alegres durante la cuarentena, sí les afectó su rendimiento académico, en cambio al $4.7 \%$ no les afectó. Por otro lado, a un $8.2 \%$ de los estudiante del grupo quienes presentaron uno de los siguientes estados: ira-hostilidad, neutro, o pasaron por todos los estados, sí les afectó su rendimiento académico, en tanto que a un 3.6\% con presencia de los tres estados de ánimo mencionados no les afectó su rendimiento académico. La prueba chi-cuadrado permitió determinar la existencia de una asociación moderada entre el estado de ánimo y la afectación del rendimiento académico ( $P$-valor $=0.338>0.05)$.

Tabla 6

Asociación entre el estado de ánimo y afectación del rendimiento académico (RA)

\begin{tabular}{|c|c|c|c|c|c|c|c|c|}
\hline & & & & \multicolumn{4}{|c|}{ Estado de ánimo durante la cuarentena } & \multirow{2}{*}{$\begin{array}{c}\text { Total } \\
\text { Todos los } \\
\text { estados }\end{array}$} \\
\hline & & & Ansiedad & $\begin{array}{c}\text { Ira- } \\
\text { hostilidad }\end{array}$ & $\begin{array}{c}\text { Tristeza- } \\
\text { Depresión }\end{array}$ & Alegría & Neutro & \\
\hline \multirow[t]{4}{*}{ RA } & $\mathrm{SI}$ & Count & 29 & 1 & 18 & 12 & 3 & 3 \\
\hline & & $\%$ of Total & $34.1 \%$ & $1.2 \%$ & $21.2 \%$ & $14.1 \%$ & $3.5 \%$ & $3.5 \%$ \\
\hline & NO & Count & 10 & 2 & 2 & 4 & 0 & 1 \\
\hline & & $\%$ of Total & $11.8 \%$ & $2.4 \%$ & $32.4 \%$ & $4.7 \%$ & $0.0 \%$ & $1.2 \%$ \\
\hline \multirow[t]{2}{*}{ Total } & & Count & 39 & 3 & 20 & 16 & 3 & 4 \\
\hline & $\%$ Total & & $45.9 \%$ & $3.6 \%$ & $23.6 \%$ & $18.8 \%$ & $3.5 \%$ & $4.7 \%$ \\
\hline
\end{tabular}

Fuente: elaboración propia.

Además, se estableció que existe una asociación leve entre la virtualidad y la afectación del rendimiento académico ( $P$-valor $=0.129>0.05)$; al respecto, al $24.4 \%$ de los estudiantes del grupo quienes si estuvieron de acuerdo con la virtualidad, ésta sí los afectó en su rendimiento académico, en cambio al $10.6 \%$ no les afectó. Por otro lado, al $55.3 \%$ de los estudiantes del grupo quienes no estuvieron de acuerdo con la virtualidad, ésta sí los afectó en su rendimiento académico, mientras que al 11.8\% no les afectó su RA. 


\section{Discusión}

Los hallazgos del presente estudio han reflejado que, con la pandemia generada por el coronavirus, la vida estudiantil universitaria también ha cambiado drásticamente, afectándose su salud física y emocional (Ortíz et al., 2020); el confinamiento (periodo de cuarentena) influyó sobre el estado de ánimo y la convivencia familiar; la percepción de los estudiantes indica que de forma moderada, su rendimiento académico fue impactado por la pandemia, las clases recibidas por medios virtuales, el estado de ánimo y la convivencia familiar. Aunque la UPTC ha promovido el uso de las TIC y de las herramientas virtuales para el aprendizaje, aún se requiere adecuar y optimizar la conectividad desde los hogares donde los estudiantes han tenido que pasar su cuarentena, de modo que, puedan interactuar sin dificultad y aprovechar los contenidos ofrecidos por el profesor universitario (Vargas, 2019).

Los resultados permitieron establecer que, los estudiantes perciben que su estado de ánimo durante la cuarentena se constituyó en un aspecto que los afectó de forma negativa en la adquisición de nuevos conocimientos relacionados con las asignaturas de su carrera profesional; también mencionaron que la convivencia en su núcleo familiar antes de la cuarentena afectaba su estado de ánimo de forma positiva, y durante el confinamiento, los afectó de forma negativa lo que influyó sobre su rendimiento académico; además, el hecho de que un $57 \%$ de los estudiantes no estuviera de acuerdo con la virtualidad en su proceso formativo y encontrarse en cuarentena también afectó su rendimiento académico de forma negativa. Por otro lado en correspondencia con el sexo, para los hombres ha sido más difícil la vida diaria en su hogar; sin embargo las mujeres mostraron más ansiedad y tristeza-depresión que los hombres; estos aspectos concuerdan con lo expresado en divesos estudios sobre andiedad y depresión (Lozano, 2020; Kang et al., 2020; OMS, 2020b; Shigemura et al., 2020).

La percepción estudiantil proporcionó indicios de que durante la cuarentena, ellos estaban preparados de forma parcial para recibir sus clases de modo virtual, desarrollar actividades de teletrabajo estdiantil, autoaprendizaje, trabajo colaborativo, distintas maneras de comunicación y realizar evaluaciones mediante estrategias propias de la educación virtual (Varguillas y Bravo, 2020; Bonilla, 2020). Durante el confinamiento, los estudiantes sintieron susto, desconcierto y hasta pánico, aumentaron sus niveles de ansiedad, depresión, tristeza y hostilidad (Munevar, Burbano \& Flórez, 2019), estos aspectos también afectaron su estado emocional y deterioraron su calidad de vida; por otra parte, con el paso del tiempo, los estudiantes pudieron controlarse y adaptarse para mejorar sus capacidades de ineteracción virtual con los contenidos de sus asignaturas y sus docentes; el proceso formativo fue mediado a través de aulas virtuales, videoconferencias, plataformas virtuales y didácticas específicas (Cardona, Ramírez \& Rivas, 2020; Burbano et al., 2020); no obstante, el trabajo desarrollado por los estudiantes fue extenuante, con altas cargas de ejercitación y evaluaciones, eventualidades que generaron desgastes tanto en la salud física como psicológica del estudiante universitario (Cleland, McKimm, Fuller, Taylor \& Janczukowicz, 2020).

De forma general, el futuro de la sociedad, la economía, la salud, la educación y las formas de interacción humana es incierto, la pandemia generada por el virus SARS-CoV-2 o Covid 19 no se ha superado y continúa siendo una amenaza de alto riesgo para la vida de las personas, llámense estudiantes, profesores, padres, hijos, trabajadores, gerentes, médicos o gobernates, ricos o pobres. En particular, la vida estudiantil continúa afectándose tanto por la pandemia como por las competencias digitales (Martínez \& Garcés, 2020), las cuales han de formar parte de su interacción cotidiana con sus profesores y el aprendizaje de sus asignaturas, en el futuro cercano.

En este contexto, se requiere del esfuerzo de todos para que esta crisis termine lo más pronto posible; hasta tanto, los científicos y los laboratorios deben seguir trabajando para producir los millones de vacunas requeridas 
para debilitar al virus en un futuro próximo; asimismo, se necesita de la disciplina, la conciencia y el ánimo de todos, incluidos los estudiantes universitarios, para asumir con responsabilidad los protocolos de bioseguridad y contener los embates de este virus que, ha cobrado miles de vidas en el territorio colombiano y en el resto de países del mundo. Por consiguiente, hay necesidad de subir el estado de ánimo, soportar con paciencia el confinamiento o el aislamiento preventivo, y sortear de la mejor manera los impactos negativos cusados por la pandemia.

\section{Conclusiones}

Este trabajo permite concluir que, los estudiantes consideran al estado de ánimo durante la cuarentena un factor que afectó de forma negativa y fuerte la adquisición de nuevos conocimientos; existe una alta relación entre la adquisición de nuevo conocimiento y el afianzamiento de conceptos previos, la convivencia en su núcleo familiar antes de la cuarentena afectaba su estado de ánimo de forma positiva, y durante la cuarentena fue afectado de forma negativa, lo cual influyó de forma relevante su rendimiento académico. Sin embargo, el confinamiento también proporcionó espacios de reflexión estudiantil para aumentar su resiliencia y mejorar sus estrategia de aprendizaje por medios virtuales para que en el futuro próximo puedan ser más competentes tanto en sus procesos de formación universitaria como en el campo laboral cuando sean profesionales.

Finalmente, se evidenció la existencia de una asociación moderada de la virtualidad, el estado de ánimo y la convivencia familiar del estudiante con su rendimiento académico. Además, los estudiantes perciben que la pandemia, el confinamiento y la implementación del trabajo virtual realizado desde el hogar, generaron impactos negativos de tipo económico, emocional y social en sus demás familiares. Este trabajo muestra la necesidad de efectuar exploraciones frecuentes y oportunas que posibiliten mejorar la situación académica del estudiante universitario para evitar su deserción durante la pandemia o después, en el transcurso de su vida universitaria.

\section{Referencias bibliográficas}

Burbano, V. M. A., Valdivieso, M. A., \& Burbano, A. (2020). El conocimiento didáctico del contenido sobre probabilidad en profesores de matemáticas de la educación básica secundaria colombiana. Espacios, 41(37), 112-125.

Bonilla, J. A. (2020). Las dos caras de la educación en el COVID-19. CienciAmérica, 9(2), 89-98. http://dx.doi.org/10.33210/ca.v9i2.294.

Cardona, C. M., Ramírez, M., \& Rivas, E. (2020). Educación superior en un mundo virtual, forzado por la pandemia del Covid 19. Espacios, 41(35), 44-57.

Caridad, M., Castellano, M., \& Cardeño, N. (2019). Integración de las tecnologías de la información y comunicación en la enseñanza universitaria: Reto dominante por alcanzar. Espacios, 40(12), 4-14.

Carreón, J. (2018). Confiabilidad y validez de un modelo de gobernanza percibida de la inseguridad. Sin Frontera, 11 (27), 1-53.

Cleland, C., McKimm, J., Fuller, R., Taylor, D. \& Janczukowicz, J. (2020). Adapting to the impact of COVID-19: Sharing stories, sharing practice. Medical Teacher, 1-4. https://doi.org/10.1080/0142159X.2020.1757635

Costa, C., \& López, X. (2020). Communication and coronavirus crisis in Spain. First lessons. El profesional de la información, 29(3), 1-18. https://doi.org/10.3145/epi.2020.may.04

Del Pino, T., Peñate, W., \& Bethencourt, J. (2010). La escala de valoración del estado de ánimo (EVEA): análisis de la estructura factorial y de la capacidad para detectar cambios en estados de ánimo. Análisis y modificación de la conducta, 36, 19-32. 
Díaz, A. L., Prados, J. S. F., Canos, V. F., \& Martínez, A. M. M. (2020). Impactos del confinamiento por el COVID19 entre universitarios: Satisfacción Vital, Resiliencia y Capital Social Online. RISE, 9(1), 79-104.

Facultad de Ciencias Sociales de la Universidad Nacional de Córdoba, a través del Programa Feminismo, Sexualidades y Derechos y la Comisión Géneros y Disidencias del Consejo Social conformada por CISCSA Red Mujer y Hábitat de América Latina y el Caribe, Las Alicias Córdoba Capital- PS, SeAP y Católicas por el Derecho a Decidir Argentina (2020). Los efectos de la pandemia COVID-19: mucho más que un problema de salud. ¿Cómo impactan las medidas de aislamiento social en los cuerpos de mujeres, lesbianas y trans en Córdoba? Recuperado el 21 de Abril de 2020, de https://rdu.unc.edu.ar/bitstream/handle/11086/15570/Los\%20efectos\%20de\%20la\%20pandemia\%20COV ID-19\%20\%20mucho\%20m\%C3\%A1s\%20que\%20un\%20problema\%20de\%20salud.pdf?sequence=2\&isAllowed=y

Johnson, M., Saletti, L., \& Tumas, N. (2020). Emotions, concerns and reflections regarding the COVID-19 pandemic in Argentina. Ciência \& Saúde Coletiva. DOI: 10.1590/1413-81232020256.1.10472020

Kang, L., Li Y., Hu S., Chen, M., Yang, C., \& Yang, B. (2020). The mental health of medical workers in Wuhan, China dealing with the 2019 novel coronavirus. Lancet Psychiatry, 7(3):e14. DOI: 10.1016/S22150366(20)30047-X

Lozano, A. (2020). Impacto de la epidemia del Coronavirus (COVID-19) en la salud mental del personal de salud y en la población general de China. Revista de Neuro-Psiquiatría, 83(1), 51-56.

Martínez, J., \& Garcés, J. (2020). Competencias digitales docentes y el reto de la educación virtual derivado de la covid-19. Educación y Humanismo, 22(39), 1-16.

Membiela, M., Sánchez, E. \& Pena, J.A. (2019). La interrelación entre el "capital humano" y el "capital social". Una aproximación al caso español. Atlantic Review of Economics (ARoEc), 2(2), 1-18.

Mendoza, H. H., Burbano, V. M., \& Valdivieso, M. A. (2019). El Rol del Docente de Matemáticas en Educación Virtual Universitaria. Un Estudio en la Universidad Pedagógica y Tecnológica de Colombia. Formación universitaria, 12(5), 51-60.

Millán, M. (2020). Trabajo social y COVID-19. Un análisis de las consecuencias sociales y sus implicaciones para la intervención social con colectivos vulnerables. Congreso internacional virtual sobre COVID-19. Consecuencias psicológicas, sociales, políticas y económicas. Recuperado el 20 de Mayo de 2020, de https://www.eumed.net/actas/20/covid/17-trabajo-social-y-covid-19-un-ana-lisis-de-las-consecuenciassociales.pdf

Mulaik, S. A. (2009). Linear Causal Modeling with Structural Equations. Boca Raton: CRC Press, Taylor and Francis Group.

Munevar, S. K., Burbano, V. M., \& Flórez, J. A. (2019). La AF como alternativa de formación para disminuir la agresividad escolar: un estudio comparativo. Revista virtual Universidad católica del norte, 56, 141-160.

Nájera, M. J., Aguayo, J. M. B., Guillén, J. C., \& Lirios, C. G. (2020). La percepción de riesgo en estudiantes universitarios ante la propagación del coronavirus SARS-COV-2 y la enfermedad COVID-19. Revista de Psicología de la Universidad Autónoma del Estado de México, 9(17), 94-107.

Ondé, D. (2017). Uso de la prueba Chi-cuadrado del modelo de independencia como fuente de evidencia empírica en Análisis Factorial Confirmatorio. Madrid: Tesis de doctorado. Universidad Autónoma De Madrid Facultad De Psicología.

Organización Mundial de la Salud -OMS- (2020a). Alocución de apertura del Director General de la OMS en la conferencia de prensa sobre la COVID-19. 7 de julio de 2020. Recuperado el 8 de Julio de 2020, de 
https://www.who.int/es/dg/speeches/detail/who-director-general-s-opening-remarks-at-the-mediabriefing-on-covid-19---7-july-2020

Organización Mundial de la Salud -OMS- (2020b). Preguntas y respuestas sobre la enfermedad por coronavirus (COVID-19). Recuperado el 18 de Julio de 2020, de https://www.who.int/es/emergencies/diseases/novelcoronavirus-2019/advice-for-public/q-a-coronaviruses

Ortíz, E. I., Herrera, E., \& De La Torre, A. (2020). Coronavirus (COVID 19) Infection in Pregnancy. Colombia Médica, 51(2), 1-7.

Pincay, J. (2018). Reflexiones sobre la accesibilidad web para el contenido educativo en los sistemas de administración de aprendizaje. Revista electrónica formación y calidad educativa, 6(1), 193-206.

Sampieri, R. H. (2018). Metodología de la investigación: las rutas cuantitativa, cualitativa y mixta. Ciudad de México: McGraw Hill.

Sandman, P. M. (1993). Responding to community outrage: Strategies for effective risk communication. AlHA.

Sanz, J. (2001). Un instrumento para evaluar la eficacia de los procedimientos de inducción de estado de ánimo: "La Escala de Valoración del Estado de Ánimo" (EVEA). Análisis y modificación de conducta, 27, 71110.

Shigemura, J., Ursano, R., Morganstein, J., Kurosawa, M., \& Benedek, D. (2020). Public responses to the novel 2019 coronavirus (2019-nCoV) in Japan: Mental health consequences and target populations. Psychiatry Clin Neurosci. DOI:10.1111/ pcn.12988.

Schor, C. (2020). Consecuencias subjetivas de la pandemia. Consejo Latinoamericano de Ciencias Sociales CLACSO- Pensar la pandemia. Observatorio social del coronavirus. Recuperado el 20 de Agosto de 2020, de https://www.clacso.org/consecuencias-subjetivas-de-la-pandemia/

Strauss, A., \& Corbin, J. (1998). Basics of qualitative research. Techniques and procedures for developing grounded theory. United Sates: Sage publications, Inc.

Sudriá, M. Andreatta, M., \& Defagó, M. (2020). Los efectos de la cuarentena por coronavirus (COVID-19) en los hábitos alimentarios en Argentina. Buenos Aires: DIAIETA

Tinoco, O. (2008). Una aplicación de la prueba Chi cuadrado con SPSS. Industrial Data, 11 (1), 73-77.

Trujillo, C. H. S. (2020). Manejo del paciente con infección por SARS-CoV-2/COVID-19. Consenso colombiano de atención, diagnóstico y manejo de la infección por SARS-CoV-2/COVID-19 en establecimientos de atención de la salud: Recomendaciones basadas en consenso de expertos e informadas en la evidencia ACIN-IETS. Infectio, 24(3), 70-88.

UNICEF Argentina (2020). "Encuesta de Percepción y Actitudes de la Población. Impacto de la pandemia COVID19 y las medidas adoptadas por el gobierno sobre la vida cotidiana” INFORME SECTORIAL: EDUCACIÓN. Argentina.

Valdivieso, M., (2011). Estadística Descriptiva. Apoyo al estudio Independiente. Contiene comandos en R. Tunja, Colombia: Editorial UPTC.

Vargas, G. (2019). Competencias digitales y su integración con herramientas tecnológicas en educación superior. Cuadernos Hospital de clínicas, 60(1), 88-94.

Varguillas, C., y Bravo, P. (2020). Virtualidad como herramienta de apoyo a la presencialidad: análisis desde la mirada estudiantil. Revista de Ciencias sociales, 26(1), 219-232.

Esta obra está bajo una Licencia Creative Commons Attribución-NoCommercial 4.0 International

(cc) BY-NC 